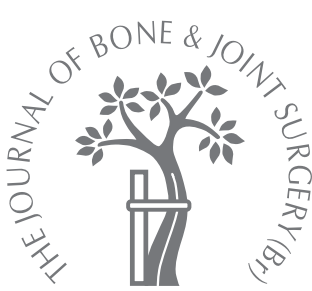

A. T. Cohen,
J. A. Skinner,
D. Warwick,
I. Brenkel

From King's College Hospital, London, England
A. T. Cohen, MSc, MD, FRACP, Consultant Vascular Physician

Department of Vascular Surgery

King's College Hospital,

Bessemer Road, London SE5 9RS, UK.

J. A. Skinner, FRCS, FRCS(Orth), Consultant Orthopaedic Surgeon Royal National Orthopaedic Hospital, Brockley Hill, Stanmore HA7 4LP, UK.

D. Warwick, MD, FRCS FRCS(Orth), Consultant Orthopaedic Surgeon University of Southampton, Tremona Road, Southampton SO16 6UY, UK

- I. Brenkel, MBChB, FRCSEd, Orthopaedic Surgeon Department of Orthopaedics Queen Margaret Hospital, Whitefield Road, Dunfermline KY12 0SU, UK.

Correspondence should be sent to $\operatorname{Dr}$ A. T. Cohen; e-mail: alexander.cohen@kcl.ac.uk

(C)2007 British Editorial Society of Bone and Joint Surgery doi:10.1302/0301-620X.89B7 $18556 \$ 2.00$

$J$ Bone Joint Surg $[\mathrm{Br}]$ 2007;89-B:887-92.

Received 21 August 2006, Accepted after revision 6 February 2006

\title{
The use of graduated compression stockings in association with fondaparinux in surgery of the hip
}

\author{
A MULTICENTRE, MULTINATIONAL, RANDOMISED, OPEN-LABEL, \\ PARALLEL-GROUP COMPARATIVE STUDY
}

Little is known about the efficacy of graduated compression stockings in preventing venous thromboembolism after hip surgery. We conducted a prospective, randomised single-blind study to determine whether the addition of compression stockings to fondaparinux conferred any additional benefit.

The study included 874 patients, of whom 795 could be evaluated (400 in the fondaparinux group and 395 in the fondaparinux plus compression stocking group). Fondaparinux was given post-operatively for five to nine days, either alone or combined with wearing stockings, which were worn for a mean 42 days (35 to 49 ).

The study outcomes were venous thromboembolism, or sudden death before day 42 . Duplex ultrasonography was scheduled within a week of day 42 . Safety outcomes were bleeding and death from venous thromboembolism.

The prevalence of deep-vein thrombosis was similar in the two groups $5.5 \%$ (22 of 400 ) in the fondaparinux group and 4.8 (19 of 395 ) in the fondaparinux plus stocking group (odds ratio $0.88,95 \%$ confidence interval 0.46 to $1.65, p=0.69$ ). Major bleeding occurred in only one patient.

The addition of graduated compression stockings to fondaparinux appears to offer no additional benefit over the use of fondaparinux alone.

Venous thromboembolism is a common complication of major surgery in the lower limb. In the absence of prophylaxis, the overall prevalence of deep-vein thrombosis (DVT) seven to 14 days after total hip replacement (THR) or surgery for hip fracture is between $50 \%$ and $60 \% .{ }^{1}$ Proximal DVT occurs in approximately $25 \%$ of patients undergoing THR and $30 \%$ of those with a hip fracture. ${ }^{1}$

Current evidence suggests that fondaparinux, a synthetic penta-saccharide, and low-dose low-molecular weight heparin (LMWH) are the most effective chemical thromboprophylactic agents ${ }^{1,2}$ for patients undergoing major hip surgery. One of these agents is usually given for five to seven days until discharge from hospital, despite the American College of Chest Physicians $(\mathrm{ACCP})^{1}$ guidelines, which recommend a minimum of ten to 14 days and up to five to six weeks after hip surgery. ${ }^{1}$ For those patients who only receive thromboprophylaxis in hospital, the prevalence of venographic DVT is $15 \%$ to $30 \%{ }^{3,4}$ at the time of discharge, and a further $10 \%$ to $25 \%^{3,4}$ will develop a new asymptomatic DVT over the following three to four weeks. 3,4
Mechanical methods of prophylaxis, such as graduated compressive stockings, intermittent pneumatic compression and foot pumps, have been poorly studied in patients undergoing surgery for hip fracture. The Cochrane Database includes 16 studies of graduated compression stockings,${ }^{5}$ only four of which are of orthopaedic patients. Of a total of 362 orthopaedic patients, ${ }^{6-}$ 9206 , none of whom had hip fractures, had been enrolled in studies which examined the effect of adding compression stockings to chemical prophylaxis. ${ }^{7,8}$

In a number of recent clinical trials the efficacy and safety of fondaparinux was tested in orthopaedic patients. ${ }^{10}$ In high-risk situations such as THR, total knee replacement (TKR) and surgery for hip fracture, post-operative fondaparinux (2.5 $\mathrm{mg}$ daily by subcutaneous injection) was shown to be more effective ${ }^{10}$ than LMWH in preventing post-operative DVT, with a comparable risk of bleeding. It was therefore the preferred method of chemical prophylaxis in this study.

Our aim was to determine whether a combination of fondaparinux and graduated compression stockings was more effective than fondaparinux alone in preventing DVT after hip surgery. 


\section{Patients and Methods}

Between January 2002 and November 2004, a multicentre, multinational, randomised, open-label phase III study was conducted in Brazil, the United Kingdom, Hong Kong and Spain in accordance with the Declaration of Helsinki, the International Conference of Harmonisation and the Good Clinical Practice guidelines.

The inclusion and exclusion criteria are shown in Table I. Patients were randomised by sealed envelope to receive fondaparinux (2.5 mg daily) for five to nine days or fondaparinux (2.5 mg daily) for five to nine days plus graduated compression stockings for 35 to 49 days which, for the purposes of this study, will be designated as the final follow-up. The compression stockings routinely used at each investigating centre were prescribed. The first dose of fondaparinux was given six hours after closure of the surgical wound and the second dose 18 to 24 hours later. Subsequent doses were administered daily at a median interval of 22 to 26 hours for between five and nine days. Long-leg stockings were used unless the thigh circumference necessitated the use of shortleg stockings. The stockings were applied pre-operatively and worn until the last follow-up visit.

Outcome measures. The primary outcome measure was the end-point of venous thromboembolism or sudden death before the last follow-up visit. Venous thromboembolism was defined by at least one of the following: objectively verified, symptomatic thromboembolism (proximal or distal DVT or fatal or non-fatal pulmonary embolism), or asymptomatic proximal DVT demonstrated by bilateral proximal ultrasound or venography. Secondary outcome measures were the components of the primary outcome and all deaths. Quality of life was measured using the EuroQoL EQ-5D questionnaire, ${ }^{11}$ which assesses a patient's perception of their quality of life. It describes health status in terms of five dimensions: mobility, self-care, usual activity, pain/discomfort and anxiety/depression. Each dimension is divided into three levels: 1) no problem; 2) some problem; 3 ) extreme problem. The highest possible health state is scored as $11111=1.0$ and the worst is $33333=-0.59$. Quality of life was measured before surgery, at the end of the treatment period, or on withdrawal from the study, and at the last follow-up visit. The safety outcomes were major and minor bleeding as adjudicated by the Critical Events Committee, the need for transfusion, death, adverse events, and serious adverse events. Major bleeding was defined as fatal bleeds; bleeding which lead to re-operation or into critical organs; clinically-overt bleeding associated with a fall in haemoglobin level of $2 \mathrm{~g} / \mathrm{dl}$ or to transfusion of two or more units, or warranting cessation of treatment. Clinically-significant bleeding was defined as non-major bleeding requiring intervention or unscheduled contact, or with patient discomfort. All other bleeding was defined as minor. The committee consisted of specialists in the field of venous thrombosis and ultrasound, and was responsible for examining the critical events reported by the investigators.
Statistical methods. Randomisation took place preoperatively and was stratified by centre, using balanced randomisation blocks. Treatment was allocated using sealed envelopes. The calculation of sample size was based on the primary outcome. It was assumed that the underlying event rate for fondaparinux alone was $9.3 \%$ and that for fondaparinux plus graduated compression stockings was $4.7 \%$ (i.e. a $50 \%$ reduction in event rate). Allowing for $10 \%$ missing the end-point, $80 \%$ power and a $5 \%$ significance level, a total sample size of 1072 patients (536 per group) was required. Outcome measures were based on the intention-to-treat population, that is, all patients who received at least one dose of study medication analysed according to the treatment to which they were randomised. For the primary outcome measure, the two groups were compared using logistic regression, adjusting for country (United Kingdom or Brazil; Hong Kong and Spain combined as a result of low patient numbers) and the type of hip surgery. Secondary outcome measures were analysed in the same way as the primary.

Summaries of the quality of life and safety outcome measures were based on the safety population (all patients who received at least one dose of study medication analysed according to the treatment they actually received).

\section{Results}

Of the 874 patients enrolled into the study, 856 (97.9\%) were randomised to treatment. The other 18 (2.1\%) either withdrew consent, did not meet all the early criteria, postponed surgery for six months or cancelled surgery, had symptomatic venous thromboembolism, the investigator decided not to randomise them, or there was a pharmacy error. There were 426 allocated to fondaparinux, and 430 to fondaparinux plus compression stockings. Of these 856 , $61 \mathrm{did}$ not receive the study medication (26 fondaparinux and 35 fondaparinux plus stockings) because they withdrew from the trial. The intention-to-treat population consisted of 400 in the fondaparinux group and 395 in the fondaparinux plus graduated compression stocking group. The safety population consisted of 404 in the fondaparinux group and 391 in the fondaparinux plus graduated compression stocking group. The flow of patients during the study is shown in Figure 1.

The demographics and risk factors of each group are shown in Table II and are comparable. The surgical history within 12 months of the study and the surgical details were also similar (Table III).

Treatment details. Fondaparinux was administered for a mean of seven days (1 to 39 ) in each group. The number of patients wearing compression stockings fell with time. For those patients in the fondaparinux plus stocking group, the median duration of application of the stockings was 44 days ( 1 to 86$)$; most $(85 \%, 323$ of 380$)$ wore them continuously until discharged. After leaving hospital, the stockings were worn continuously by $76 \%$ (252 of 330). Long-leg stockings 


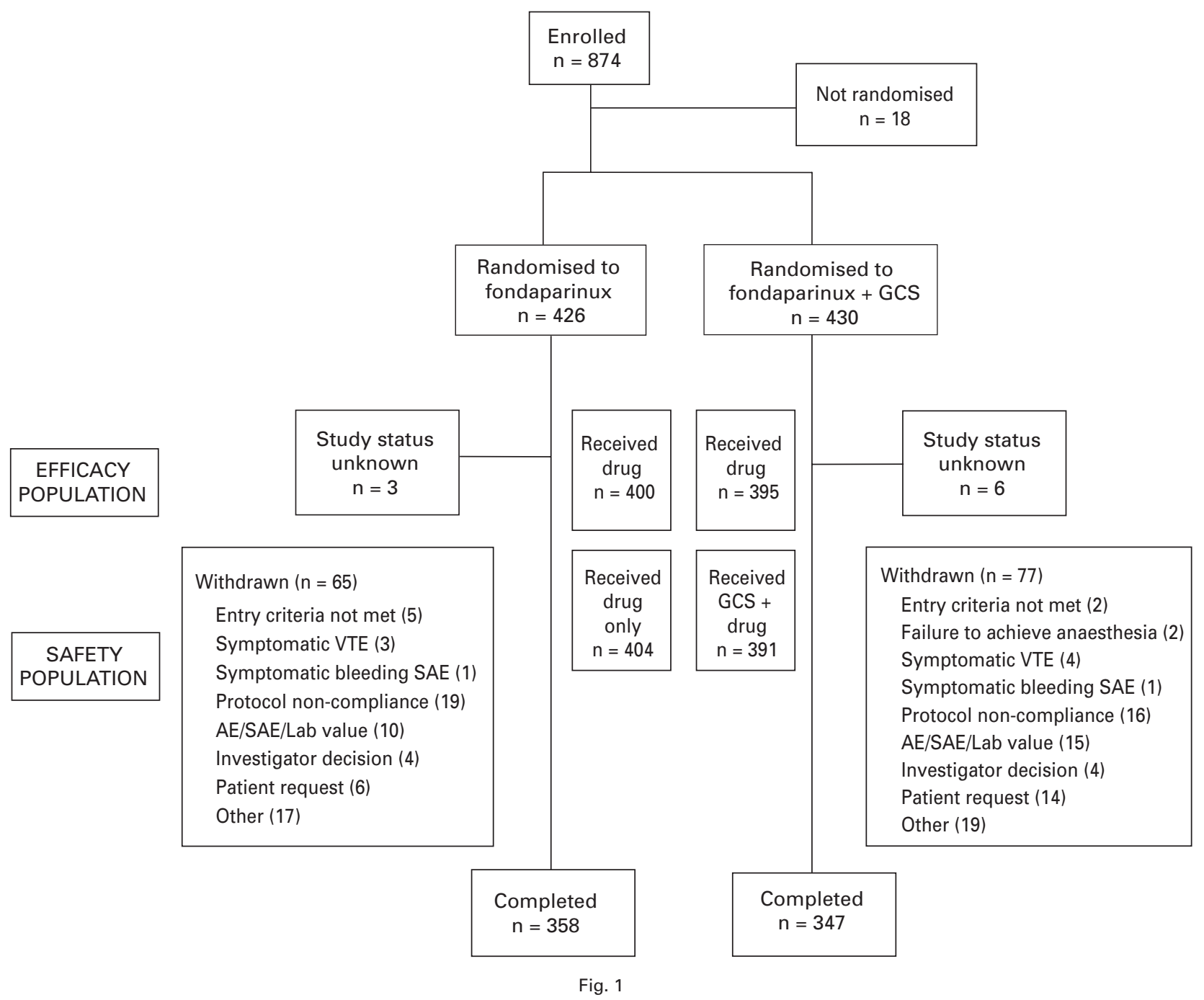

Flow of patients through the study (VTE, venous thromboembolism; SAE, serious adverse event; AE, adverse event; GCS, graduated compression stockings).

were used by $68 \%$ (266 of 389 ) and the short-leg version by the rest.

Efficacy outcome. The outcome measures (Table IV) were recorded for a similar proportion of patients in the two groups. No patient in either group suffered a fatal pulmonary embolism or sudden death by the time of their last follow-up. The rates of venous thromboembolism for longleg and short-leg stockings were $5.6 \%$ (14 of 251 ) and $4.2 \%$ (5 of 118), respectively.

Safety outcomes. The safety outcomes are recorded in Table V. Only one patient $(<1 \%)$ had a major episode of bleeding. Overall, 2\% (7 of 391) of patients had adverse events related to the wearing of stockings.

Three patients in the fondaparinux group died from causes unrelated to thromboembolism and one in the fondaparinux plus stocking group. The causes of death were cardiac failure, haemoptysis, ischaemic heart disease and myocardial infarction. A similar proportion of patients in the fondaparinux group ( $8 \%, 32$ of $404 ; 37$ events) and the fondaparinux plus stocking group $(7 \%, 29$ of $391 ; 36$ events) reported serious adverse events as a result of treatment. The most frequently reported events were abdominal pain, cellulitis, constipation, dislocation of a joint prosthesis after hip arthroplasty, myocardial infarction, peripheral oedema, wound infection and superficial wound discharge. Quality of life measures. Both groups showed similar increases in health state scores and overall health status assessments (visual analogue scale) from screening to the last day of treatment and through to follow-up (Table VI).

\section{Discussion}

This is by far the largest randomised study of thromboprophylaxis and graduated compression stockings to date. Compression stockings were worn for a full six 
Table I. Inclusion and exclusion criteria

Inclusion criteria

Minimum age 18 years

Primary or revision total hip replacement

Surgery for fracture of the proximal third of the femur

Exclusion criteria

Bilateral joint surgery

Multiple trauma

Delay $>24$ hours between trauma and admission

Conditions precluding use of graduated compression stockings

Leg oedema

Peripheral vascular disease

Peripheral neuropathy

Marked leg deformity

Conditions that increase the risk of bleeding

Pregnant/lactating women or those of child bearing age taking inadequate contraceptive precautions

Table II. Demographics and medical and surgical risk factors

\begin{tabular}{|c|c|c|}
\hline & Fondaparinux ( $n=404$ ) & Fondaparinux + GCS $^{*}(n=391)$ \\
\hline Number with data & 403 & 390 \\
\hline Mean age (range) & 65 (23 to 99$)$ & 65 (18 to 97$)$ \\
\hline \multicolumn{3}{|l|}{ Gender } \\
\hline Female $(\%)$ & $224(56)$ & $228(58)$ \\
\hline Mean body mass index (range) & 28 (16.9 to 50.1$)$ & 28 (15.0 to 44.6$)$ \\
\hline Obesity (\%) & 85 of $402(21)$ & 75 of $388(19)$ \\
\hline History of $\mathrm{VTE}^{\dagger}(\%)$ & $13(3.2)$ & $11(2.8)$ \\
\hline Family history of VTE (\%) & $12(2.9)$ & $16(4.1)$ \\
\hline History of cancer $(\%)$ & $29(7.2)$ & $18(4.6)$ \\
\hline Varicose veins and/or chronic venous insufficiency (\%) & $26(6.5)$ & $37(9.5)$ \\
\hline Previous ischaemic heart disease $(\%)$ & $14(3.4)$ & $20(5.1)$ \\
\hline Previous stroke (\%) & $7(1.7)$ & $3(0.8)$ \\
\hline Chronic heart failure (\%) & $3(0.7)$ & $4(1.0)$ \\
\hline Chronic respiratory failure $(\%)$ & $7(1.7)$ & $2(0.5)$ \\
\hline Personal history of thrombophilia (\%) & $0(0)$ & $1(0.3)$ \\
\hline Oestrogen use (\%) & $13(3.2)$ & $16(4.0)$ \\
\hline
\end{tabular}

* GCS, graduated compression stockings

† VTE, venous thromboembolism

Table III. Surgical details

\begin{tabular}{|c|c|c|}
\hline & Fondaparinux $(n=404)$ & Fondaparinux $+\mathrm{GCS}^{*}(\mathrm{n}=391)$ \\
\hline Number with data available & 403 & 390 \\
\hline Previous hip replacement in last 12 months (\%) & $22(5.4)$ & $16(4.1)$ \\
\hline Previous knee replacement in last 12 months (\%) & $0(0)$ & $0(0)$ \\
\hline Previous hip fracture in last 12 months (\%) & $2(0.5)$ & $1(0.2)$ \\
\hline Elective $\mathrm{THR}^{\dagger}(\%)$ & $381(94.5)$ & $375(96.1)$ \\
\hline Primary & $362(95)$ & $352(94)$ \\
\hline Revision & $19(9)$ & $23(6)$ \\
\hline Standard fracture surgery (\%) & $23(5.7)$ & $16(4.1)$ \\
\hline Cervical & $17(74)$ & $9(56)$ \\
\hline Trochanteric & $6(26)$ & $7(44)$ \\
\hline
\end{tabular}

* GCS, graduated compression stockings

† THR, total hip replacement

weeks, and yet there was no evidence of added benefit over thromboprophylaxis with fondaparinux only. The prevalence of venous thromboembolism was similar whether patients wore graduated compression stockings or not: $5.8 \%$ in the fondaparinux group and $5.1 \%$ in the fondaparinux plus stockings group; the odds ratio was $0.88(95 \%$ confidence interval $(\mathrm{CI}) 0.46$ to $1.65, \mathrm{p}=$ $0.69)$. There is therefore no advantage in using gradu- 
Table IV. Outcome measures, intention-to-treat (all patients) and total hip replacement (THR) patients only

\begin{tabular}{|c|c|c|c|}
\hline & $\begin{array}{l}\text { Fondaparinux } \\
(\mathrm{n}=\mathbf{4 0 0 )}\end{array}$ & $\begin{array}{l}\text { Fondaparinux plus GCS * } \\
(n=395)\end{array}$ & $\begin{array}{l}\text { Adjusted odds ratio }{ }^{\dagger} \\
(95 \% \mathrm{Cl})\end{array}$ \\
\hline VTE $^{\ddagger}$ or sudden death by day 42 (all patients) (\%) & $22(5.5)$ & $19(4.8)$ & $0.88(0.46$ to 1.65$) p=0.69^{\S}$ \\
\hline Asymptomatic proximal DVT by day $42(\%)$ & $19(4.8)$ & $16(4.1)$ & $0.86(0.43$ to 1.70$)$ \\
\hline Both asymptomatic and symptomatic VTE together (\%) & $1(0.3)$ & $1(0.3)$ & \\
\hline Symptomatic VTE (\%) & $4(1)$ & $4(1)$ & \\
\hline Asymptomatic proximal or symptomatic distal DVT by day $42(\%)$ & 5.0 & $16(4.1)$ & $0.86(0.43$ to 1.70$)$ \\
\hline $\begin{array}{l}\text { Proximal DVT or fatal or non-fatal pulmonary embolism by day } 42 \\
(\%)\end{array}$ & $19(4.8)$ & $16(4.1)$ & $0.86(0.43$ to 1.70$)$ \\
\hline VTE or sudden death by day 42 (THR patients only) (\%) & 20 of $363(5.5)$ & 10 of $358(5.3)$ & $0.96(0.50$ to 1.83$)$ \\
\hline VTE-related deaths (\%) & $0(0)$ & $0(0)$ & \\
\hline
\end{tabular}

* GCS, graduated compression stockings

$\dagger$ adjusted for country (Brazil, Hong Kong and Spain combined in one group and United Kingdom was the other group) and type of surgery (hip fracture or hip replacement)

¥ VTE, venous thromboembolism

$\S p$-value only given for primary efficacy outcome (other analyses all other outcomes were also non-significant)

I DVT, deep-vein thrombosis

Table V. Safety outcomes and the most frequent adverse events $(\geq 10 \%$ of patients)

\begin{tabular}{|c|c|c|c|}
\hline & $\begin{array}{l}\text { Fondaparinux } \\
(n=404)\end{array}$ & $\begin{array}{l}\text { Fondaparinux + GCS } \\
(\mathrm{n}=391)\end{array}$ & $\begin{array}{l}\text { Percentage difference } \\
\left(95 \% \mathrm{CI}^{\dagger}\right)\end{array}$ \\
\hline Major bleeding (\%) & $1(0.2)$ & $0(0)$ & $0.25(-0.49$ to 0.98$)$ \\
\hline Minor bleeding (\%) & $29(7.1)$ & $25(6.3)$ & $0.78(-2.96$ to 4.53$)$ \\
\hline Clinically significant minor bleeding (\%) & $20(4.9)$ & $16(4.1)$ & $0.86(-2.28$ to 4.00$)$ \\
\hline Need for transfusion (\%) & $134(33.1)$ & $141(36.1)$ & 2.89 (-3.97 to 9.76$)$ \\
\hline Anaemia (\%) & $81(20)$ & $93(23.7)$ & $3.74(-2.27$ to 9.74$)$ \\
\hline Wound secretion (\%) & $59(14.6)$ & $56(14.3)$ & $0.28(-4.86$ to 5.42$)$ \\
\hline Haemoglobin decreased (\%) & 48 (11.9) & $43(10.9)$ & $0.88(-3.79$ to 5.56$)$ \\
\hline Non-VTE ${ }^{\ddagger}$ related deaths $(\%)$ & $3(0.7)$ & $1(0.7)$ & $0.49(-0.74$ to 1.71$)$ \\
\hline
\end{tabular}

ated compression stockings for up to six weeks if fondaparinux is given.

Stockings are used by approximately $70 \%$ of patients in the United Kingdom, whereas in Europe and the USA many orthopaedic surgeons recommend their use for four to six weeks. ${ }^{1}$ They have been recommended for patients at all levels of risk. ${ }^{12}$ Compliance may be a problem and the stockings can cause complications, as was seen in $2 \%$ of the patients in this study, a significant figure, considering that the patients were carefully selected to avoid such complications. Major leg ischaemia has been described after both hip and knee replacement. ${ }^{13}$ The stockings are timeconsuming to measure and fit, disliked by patients, inconvenient and costly. ${ }^{14,15} \mathrm{~A}$ recent study in frail patients in a palliative care setting showed that stockings were unaccept- able and that parenteral anticoagulation improved the patient's overall quality of life. ${ }^{15}$

This study has a number of strengths, including its large sample size. It was well randomised and the level of compliance was high. However, the study was stopped early because the differences between the groups were so small that it would have been futile to continue. The main weakness was the need to use stockings in an 'open' fashion as it was impossible to blind this intervention with sham stockings. The study was designed to reduce this weakness by blinding the ultrasonographers to the treatment. The use of ultrasound to diagnose DVT in this setting has not been widely tested but it is known to be less sensitive than venography. Venography, however, is invasive and has a significantly higher failure rate than ultrasound. Although ultrasound is less sensitive, the measurement 
Table VI Quality of life EQ-5D (all patients in the safety sample population)

\begin{tabular}{lll}
\hline & $\begin{array}{l}\text { Fondaparinux } \\
\text { (n= 404) }\end{array}$ & $\begin{array}{l}\text { Fondaparinux + GCS* } \\
\text { (n= 391) }\end{array}$ \\
\hline $\begin{array}{l}\text { Health state scores: median (range) (n) } \\
\quad \text { Screening }\end{array}$ & $0.21(-0.59$ to 1.00$)(394)$ & $0.16(-0.59$ to 1.00$)(377)$ \\
$\quad$ Last day of treatment & $0.59(-0.59$ to 1.00$)(344)$ & $0.59(-0.43$ to 1.00$)(345)$ \\
$\quad$ Follow-up & $0.76(-0.17$ to 1.00$)(328)$ & $0.71(-0.09$ to 1.00$)(330)$ \\
& & \\
Overall health status: median (range) (n) & $65(0$ to 100) (389) & $60(0$ to 100 (369) \\
$\quad$ Screening & $70(20$ to 100$)(339)$ & $70(6$ to 100$)(341)$ \\
$\quad$ Last day of treatment & $80(0$ to 100) (325) & 80 (3 to 100) (333) \\
$\quad$ Follow-up & &
\end{tabular}

of the relative risk reduction, the key outcome, does not seem to be affected. ${ }^{16}$

The two previous high-quality studies examining the addition of compression stockings to pharmacological methods showed a $65 \%$ reduction of relative risk in the stocking group. ${ }^{5,7,8}$ However, these studies had less than one-third of the number of patients included in our study. Why did our study not show a difference between the two methods of treatment? The possible explanations include a lack of effect of the stockings, a lack of effect of the stockings when used with a very effective anticoagulant, a chance finding (unlikely given the sample size), poor compliance, inappropriate or incorrect use (unlikely as nursing staff were regularly encouraged to ensure that the stockings were properly applied and used), and poor sensitivity of the study (unlikely, given the sample size and event rates). The rates of symptomatic venous thromboembolism were similar and very low in both groups, consistent with the known efficacy of fondaparinux. A large study of patients with hip fractures has shown that extended prophylaxis for five weeks results in a highly significant reduction in asymptomatic and symptomatic venous thromboembolism. ${ }^{17}$

The addition of graduated compression stockings does not appear to improve the effectiveness of prophylactic anticoagulation with fondaparinux. As graduated compression stockings are time-consuming to measure and fit, inconvenient, and expensive, we recommend that their use in hip surgery be reconsidered. In future, their use may be replaced by a more extended period of anticoagulation.

\section{Supplementary Material}

$\because$ A further opinion by Mr G. C. Bannister is available with the electronic version of this article on our website at www.jbjs.org.uk

The author or one or more of the authors have received or will receive benefits for personal or professional use from a commercial party related directly or indirectly to the subject of this article.

\section{References}

1. Geerts WH, Pineo GF, Heit JA, et al. Prevention of venous thromboembolism. Chest 2004;126:338-400.

2. Bjornara BT, Gudmundsen TE, Dahl OE. Frequency and timing of clinical venous thromboembolism after major joint surgery. J Bone Joint Surg [Br]2006;88-B:386-91.

3. Planes A, Vochelle N, Darmon JY, et al. Risk of having deep-venous thrombosis after hospital discharge in patients having undergone total hip replacement: doubleblind randomised comparison of enoxaparin versus placebo. Lancet 1996;348:224-8.

4. Dahl OE, Andreassen G, Aspelin T, et al. Prolonged thromboprophylaxis following hip replacement surgery: results of a double-blind, prospective, randomised, placebo, controlled study with dalteparin (Fragmin). Thromb Haemost 1997;77:26-31.

5. Amaragiri SV, Lees TA. Elastic compression stockings for prevention of deep vein thrombosis (Cochraine Review). In: The Cochrane Library, Issue 2, 2003.

6. Barnes RW, Brand RA, Clarke W, Hartley N, Hoak JC. Efficacy of graded-compression antiembolism stockings in patients undergoing total hip arthroplasty. Clin Orthop 1978;132:61-7.

7. Ohlund C, Fransson S-V, Starck S-A. Calf compression for prevention of thromboembolism following hip surgery. Acta Orthop Scand 1983;54:896-9.

8. Fredin H, Bergqvist D, Cederholm C, Lindbald B, Nyman U. Thromboprophylaxis in hip arthroplasty: dextran with graded compression or pre-operative dextran compared in 150 patients. Acta Orthop Scand 1989;60:678-81.

9. Hui AC, Heras-Palou C, Dunn I, et al. Graded compression stockings for prevention of deep-vein thrombosis after hip and knee replacement. J Bone Joint Surg [Br] 1996;78-B:550-4.

10. Turpie AGG, Bauer KA, Eriksson BI, Lassen MR. Fondaparinux vs enoxparin for the prevention of venous thromboembolism in major orthopedic surgery: a metalanalysis of 4 randomized double-blind studies. Arch Intern Med 2002;162:1833-40.

11. Rabin R, de Charro F. EQ - SD: a measure of health status from the Euro Qol Group. Ann Med 2001;33:337-43.

12. Caprini JA, Arcelus JI, Hasty JH, Tamhane AC, Fabrega F. Clinical assessment of venous thromboembolic risk. Semin Thromb Haemost 1991;117(Suppl 3):304-12.

13. Heath DI, Kent SJS, Johns DL, Young TW. Arterial thrombosis associated with graduated pressure antiembolic stockings. Br Med J (Clin Res Ed) 1987;295:580.

14. Ingram JR. A review of thigh-length vs knee-length antiembolism stockings. $B r \mathrm{~J}$ Nurs 2003;12:845-51.

15. Noble SIR, Nelson A, Turner C, Finlay IG. Acceptability of low molecular weight heparin thromboprophylaxis for inpatients receiving palliative care: qualitative study. BMJ 2006;332:577-80.

16. Leizorovicz A, Cohen AT, Turpie AGG, et al. A randomised placebo-controlled trial of dalteparin for the prevention of venous thromboembolism in acutely ill medical patients. Circulation 2004;110:874-9.

17. Eriksson BI, Lassen MR. Duration of prophylaxis against venous thromboembolism with fondaparinux after hip fracture surgery. Arch Intern Med 2003;163:133742. 